\title{
A Rare Presentation of Central Nervous System Melioidosis
}

\author{
Shin-Shen Yong ${ }^{*}$, Siti-Aisah Hassan², Phing-Sue Wong ${ }^{1}$, Kar-Yaw Yoong1 \\ ${ }^{1}$ Department of Internal Medicine, Hospital Sultan Ismail, Johor, Malaysia \\ ${ }^{2}$ Department of Radiology, Hospital Sultan Ismail, Johor, Malaysia \\ Email: *emyong5@hotmail.com
}

How to cite this paper: Yong, S.-S., Hassan, S.-A., Wong, P.-S. and Yoong, K.-Y. (2017) A Rare Presentation of Central Nervous System Melioidosis. Open Access Library Journal, 4: e3346.

http://dx.doi.org/10.4236/oalib.1103346

Received: December 30, 2016

Accepted: January 17, 2017

Published: January 20, 2017

Copyright @ 2017 by authors and Open Access Library Inc.

This work is licensed under the Creative Commons Attribution International License (CC BY 4.0).

http://creativecommons.org/licenses/by/4.0/ (c) (i) Open Access

\begin{abstract}
We report a case of an 18-year-old army man who was diagnosed with cerebral melioidosis with disseminated abscesses. The patient presented with fever and focal neurological signs with positive cerebral imaging findings followed by a positive blood culture for Burkholderia pseudomallei. Further imaging of other sites also revealed multiple abscesses. He responded well to an intensive course of antibiotic therapy of eight-week duration followed by 6 months of eradication therapy. The repeat cerebral imaging showed complete resolution of basal ganglia abscess at the end of intensive antibiotic therapy.
\end{abstract}

\section{Subject Areas \\ Infectious Diseases}

\section{Keywords}

Melioidosis, Basal Ganglia, Abscess, Antibiotic

\section{Introduction}

Melioidosis is endemic in Southeast Asia and northern Australia and it is caused by Burkholderia pseudomallei [1]. It gains entry to the human host through skin abrasion, inhalation or ingestion. The clinical manifestations of melioidosis are variable and it commonly involves the lungs, visceral organs and occasionally musculoskeletal system with cerebral involvement being a rare presentation. The definite diagnosis is made by positive culture of the organism from blood or tissue biopsy. In endemic areas where the B. pseudomallei is detected in the blood culture or biopsy, the suspicion for systemic melioidosis should be raised.

Cerebral melioidosis are rarely reported and cases with disease presentations secondary to cranial abscesses are exceedingly rare [2]. The radiological features 
of cerebral melioidosis are variable and usually present as encephalitis or abscesses. Basal ganglia abscess is reported to be rare in central nervous system melioidosis. The duration of treatment depends on the site and extent of the infection. Prolonged intravenous (IV) therapy for four to eight weeks is needed for complicated infections such as cerebral melioidosis followed by eradication therapy.

\section{Case Presentation}

An 18-year-old army man presented with intermittent fever for one week, associated with headache, chills and occasional dry cough. He was not known to have any previous medical illness. On physical examination, his Glasgow Coma Scale (GCS) score was full. However he appeared lethargic. He was febrile with a temperature of 38.7 degree Celsius. There was no significant abnormality detected on the cardiovascular, respiratory and abdominal examination. Neurological examination revealed upper motor neuron signs on the left lower limb with hypertonia, brisk knee and ankle reflexes, decreased in motor power to 4(moving against slight resistance) with sustained ankle clonus and positive Babinski response. Neck stiffness and positive Brudzinski's sign were present. Preliminary blood investigations showed leukocytosis, white cell count of $12.7 \times$ $10^{3} / \mathrm{UL}$; and mild elevation of liver enzymes, aspartate aminotransferase of 250 $\mathrm{u} / \mathrm{L}$ and alanine aminotransferase of $171 \mathrm{u} / \mathrm{L}$ (Table 1 ). The renal function test was within normal limits and blood and urine cultures sent for cultures. The chest radiograph was reported to be normal. In view of the history of fever with significant neurological deficit, an urgent plain computed tomography (CT) brain was done.

Immediate CT brain showed an ill-defined hypodense lesion at the right basal ganglia (Figure 1). He was then treated as for meningitis with IV ceftriaxone and IV acyclovir. Lumbar puncture (LP) was performed and the cerebrospinal fluid (CSF) sent for analysis. The CSF was reported acellular, clear in appearance with elevated protein level and low glucose level suggestive of bacterial meningitis. The CSF culture and gram staining showed no organisms. Magnetic resonance Imaging (MRI) of the brain with gadolinium contrast was done which showed a ring-enhancing lesion in the right basal ganglia with subdural effusion (Figure 2). Viral screening including Hepatitis B, C and Human-immunodeficiency Virus (HIV) were all non-reactive. He remained febrile on day 4 of admission and the blood culture grew Burkholderia pseudomallei. Bedside ultrasound of the abdomen revealed splenic micro-abscesses (Figure 3). A subsequent contrast enhanced CT of the thorax, abdomen and pelvis showed airspace opacities in the right lung, left lung consolidation and multiple splenic micro-abscesses (Figure 4).

The therapy was switched to the recommended antibiotic regime according to the Royal Darwin Melioidosis Guideline [3] that includes eight weeks of intensive phase of IV meropenem followed by six months of eradication therapy with trimethoprim-sulfamethoxazole and folic acid. At the end of the treatment, he 
Table 1. Laboratory investigations.

\begin{tabular}{|c|c|c|c|}
\hline Variables & Normal range & On admission & $\begin{array}{c}\text { After 2-weeks } \\
\text { interval }\end{array}$ \\
\hline Hematocrit & $40 \%-54 \%$ & 28.2 & 36.9 \\
\hline Hemoglobin & $10-18 \mathrm{~g} / \mathrm{dL}$ & 9.3 & 12.4 \\
\hline White-cell count & $4-11 \times 10^{3} / \mathrm{UL}$ & 12.7 & 6.9 \\
\hline \multicolumn{4}{|l|}{ Differential count } \\
\hline Neutrophils & $(2.9-7.9) \times 10^{3} / \mathrm{UL}$ & 8.7 & 3.3 \\
\hline Lymphocytes & $(1.8-4.0) \times 10^{3} / \mathrm{UL}$ & 1.3 & 2.6 \\
\hline Monocytes & $(0.0-80.0) \times 10^{3} / \mathrm{UL}$ & 0.7 & 0.4 \\
\hline Eosinophils & $(0.4-2.1) \times 10^{3} / \mathrm{UL}$ & 0.0 & 0.48 \\
\hline Basophils & $(0.0-0.2) \times 10^{3} / \mathrm{UL}$ & 0.0 & 0.0 \\
\hline Platelet count & $(150-400) \times 10^{3} / \mathrm{UL}$ & 220 & 396 \\
\hline C-reactive protein & $0.0-5.0 \mathrm{mg} / \mathrm{L}$ & 218.2 & 1.70 \\
\hline Potassium & $3.5-5.0 \mathrm{mmol} / \mathrm{L}$ & 3.4 & 3.6 \\
\hline Urea & $1.7-8.3 \mathrm{mmol} / \mathrm{L}$ & 5.0 & 3.5 \\
\hline Creatinine & $62-106 \mu \mathrm{mol} / \mathrm{L}$ & 69 & 89 \\
\hline Glucose & $3.1-6.4 \mathrm{mmol} / \mathrm{L}$ & 4.4 & \\
\hline Bilirubin & $<17 \mu \mathrm{mol} / \mathrm{L}$ & 37 & 7 \\
\hline Direct & $0-5 \mu \mathrm{mol} / \mathrm{L}$ & 22 & 1 \\
\hline Protein & $66-87 \mathrm{~g} / \mathrm{L}$ & 60 & 85 \\
\hline Albumin & $34-48 \mathrm{~g} / \mathrm{L}$ & 17 & 40 \\
\hline Aspartate aminotransferase & $0-38 \mathrm{u} / \mathrm{L}$ & 250 & 21 \\
\hline Alanine aminotransferase & $<41 \mathrm{u} / \mathrm{L}$ & 171 & 42 \\
\hline Alkaline phosphatase & $0-129 \mathrm{u} / \mathrm{L}$ & 153 & 100 \\
\hline Lactate Dehydrogenase & $313-512 \mathrm{u} / \mathrm{L}$ & 1548 & \\
\hline Creatine Kinase & $39-308 \mathrm{u} / \mathrm{L}$ & 1748 & \\
\hline $\mathrm{HbA1C}$ & & 5.8 & \\
\hline \multicolumn{4}{|l|}{ CSF Biochemistry } \\
\hline Appearance & & Clear & \\
\hline CSF Glucose & $2.22-3.90 \mathrm{mmol} / \mathrm{L}$ & 2.10 & \\
\hline CSF Protein & $0.15-0.45 \mathrm{~g} / \mathrm{L}$ & 0.45 & \\
\hline CSF Albumin & $\mathrm{g} / \mathrm{L}$ & 0.19 & \\
\hline CSF Culture & & No growth & \\
\hline CSF Cell count & $\mathrm{Wbc} / \mathrm{mL}$ & Nil & \\
\hline CSF Gram stain & \multicolumn{3}{|c|}{ No organism seen } \\
\hline CSF Acid Fast Bacilli culture & \multicolumn{3}{|c|}{ Negative } \\
\hline $\begin{array}{c}\text { CSF Herpes Simplex Polymerase Chain } \\
\text { Reaction }\end{array}$ & \multicolumn{3}{|c|}{ Not detected } \\
\hline CSF Toxoplasma antibody IgM & \multicolumn{3}{|c|}{ Not detected } \\
\hline CSF Toxoplasma antibody IgG & \multicolumn{3}{|c|}{ Reactive } \\
\hline CSF Mycobacterium Tuberculosis PCR & \multicolumn{3}{|c|}{ Not detected } \\
\hline
\end{tabular}




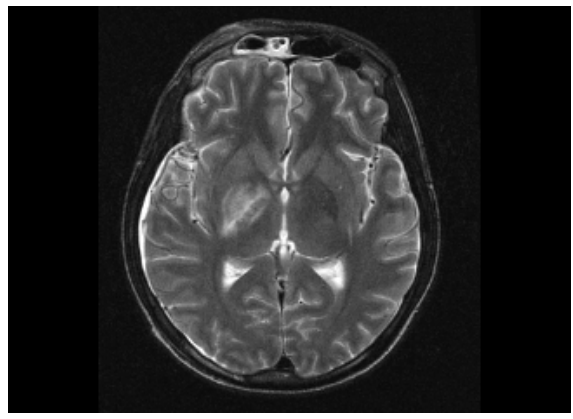

Figure 1. Non-enhanced CT brain shows hypodense lesion in the right basal ganglion.

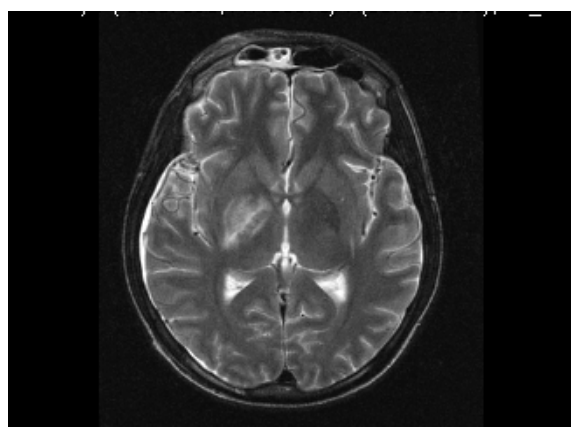

Figure 2. Axial T2WI: High signal changes in the right basal ganglion with right subdural effusion.

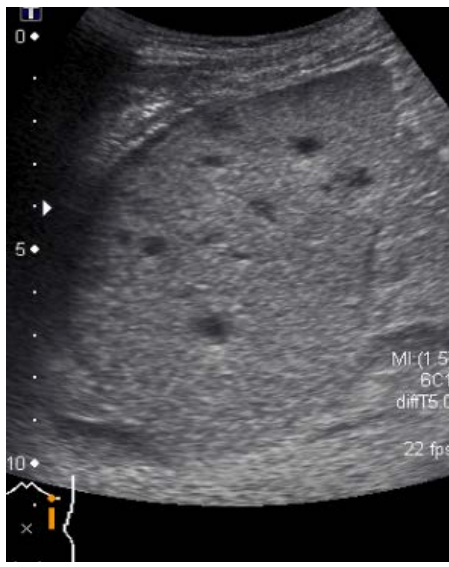

Figure 3. Splenic micro-abscesses on abdomen ultrasound.

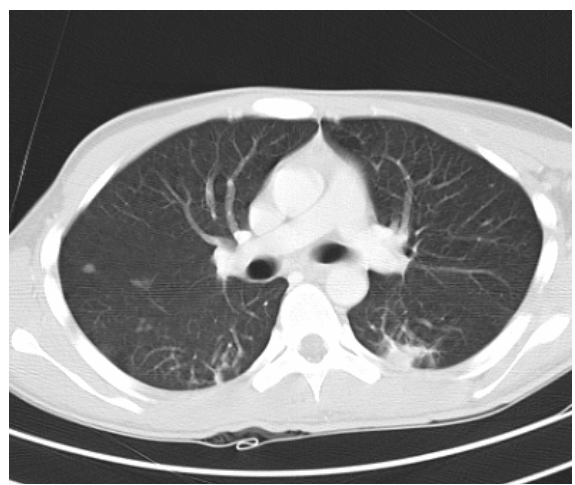

Figure 4. CT Thorax in lung setting showed air space opacities in the right lung with consolidation in the left lower lobe of the lung. 
had gained full neurological recovery and the repeated CT scan after eight weeks of treatment showed complete resolution of the previous basal ganglion lesion and subdural effusion.

\section{Discussion}

Melioidosis is an infectious disease caused by Burkholderia pseudomallei, which is endemic in the tropics [4]. It is a Gram-negative aerobic bacillus that gains entry to human host through skin abrasion, inhalation and ingestion. It has a predilection for multi-organ involvement and frequently involves the lungs, visceral organs, skin or soft tissue and rarely, meninges and the cerebrum. It has a variable clinical manifestation with non-specific radiological features and it requires a high index of clinical suspicion. The definitive diagnosis is made by presence of positive culture of the organism from blood or tissue [5]. Nevertheless, advanced imaging plays a pivotal role in establishing the extent of the disease, characterizing the lesion, making management decision, as well as following up on the disease progression.

The initial plain CT scan in this patient revealed a hypodense lesion in the right basal ganglion, leading to a possible diagnosis of encephalitis. MRI with gadolinium contrast was used to further delineate the lesion and revealed a solitary ring-enhancing lesion in the right basal ganglia with surrounding edema (Figure 5). Diffusion weighted imaging with apparent diffusion coefficient (ADP) mapping showed a central core of restricted diffusion, which is suggestive of an abscess (Figure 6 and Figure 7) [6]. MR Imaging is sensitive in delineating the high signal changes of the cortex, basal ganglia, thalamus, cerebellum and midbrain while to rule out demyelination, abscess or tumour. Central nervous system melioidosis is rarely reported and mostly presents as meningoencephalitis with cerebral abscesses and brain stem involvement which can present as cranial nerve palsies, or flaccid paraparesis alone [7]. The biochemistry findings of the CSF from the LP and the presence of Burkholderia pseudomallei in serial blood cultures confirm the diagnosis of cerebral melioidosis with disseminated infection. The CSF analysis from patients with cerebral melioidosis often shows no organism on Gram staining, leukocytosis with mononuclear cell predominance, high protein level, and normal glucose level [8] [9].

Basal ganglia are rare location for abscess formation. In adults, hematogenous brain abscesses usually occur at areas supplied by middle cerebral artery and very occasionally, in the deep tissues, such as basal ganglia and thalamus [10]. Streptococci and anaerobes are the most commonly encountered microorganisms in basal ganglia and thalamic abscesses [11]. Among the immunocompromised patients, basal ganglia is more commonly affected by toxoplasmosis and tuberculosis. Other differential diagnosis of a ring-enhancing lesion in the basal ganglia include glioblastoma, metastasis, and pyogenic abscess, subacute ischemic infarction, resolving hematoma, and demyelinating disease [12].

Cerebral melioidosis is a rare manifestation of the infection and associated with melioidosis at other sites in up to $10 \%$ of cases [8]. Therefore, the findings 


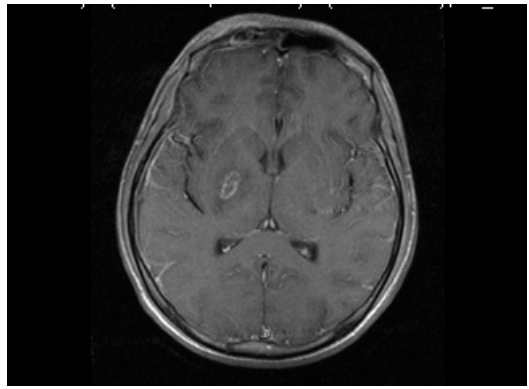

Figure 5. Post Gadolinium axial T1WI ring-enhancing lesion in the right basal ganglion.

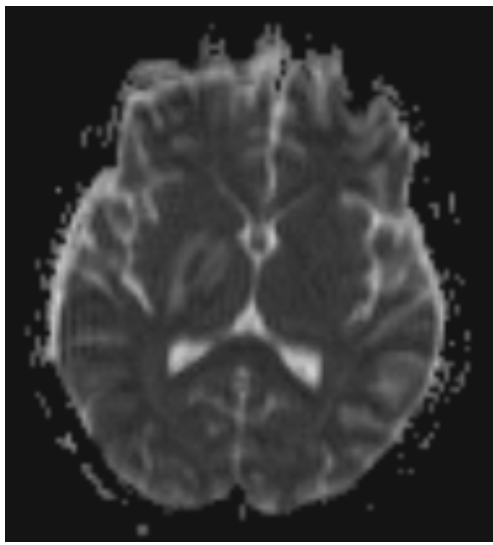

Figure 6. Diffusion weighted image $\left(4100 / 119\right.$, b value of $\left.1000 \mathrm{~s} / \mathrm{mm}^{2}\right)$ shows high signal intensity, with low signal on ADC map represent restricted diffusion of the abscess core.

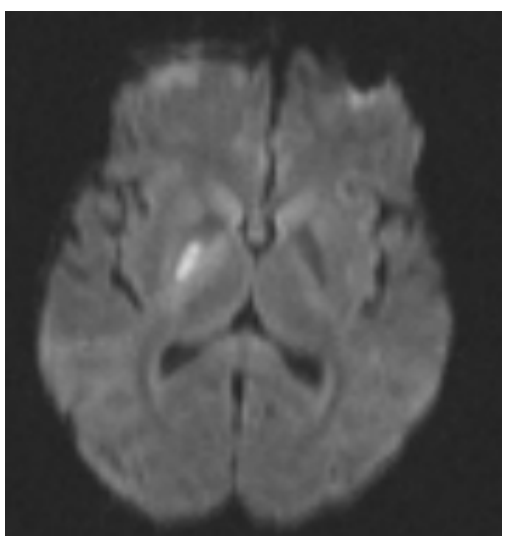

Figure 7. Diffusion weighted image $\left(4100 / 119\right.$, b value of $\left.1000 \mathrm{~s} / \mathrm{mm}^{2}\right)$ shows high signal intensity, with low signal on ADC map represent restricted diffusion of the abscess core.

of cerebral melioidosis should prompt the clinicians and radiologists to look for other foci of infection. The ultrasound is sensitive in detection of visceral microabscesses but whole body CT scan provides a good information on the extent of the disease. The CT thorax is more sensitive and shows various manifestations of melioidosis infection in the lungs, from air space opacities and nodules, to cavitating lesions and consolidation, which may not be seen on the chest $\mathrm{X}$ ray.

In this case illustration, the patient presented with symptoms of meningoencephalitis initially. The subsequent discovery of Burkholderia pseudomallei in the blood culture prompted the clinicians to look for disseminated abscesses in 


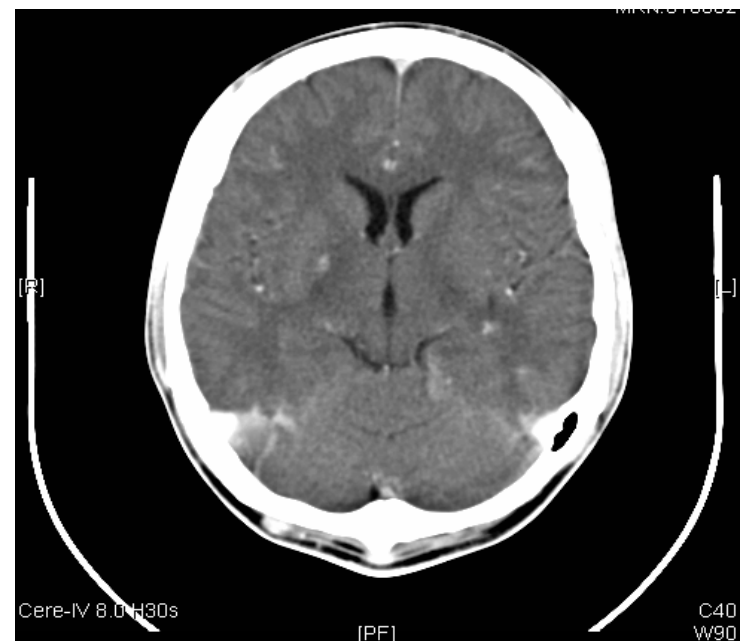

Figure 8. Post treatment CECT brain at 3-month interval: Smaller ring enhancing lesion $(2.3 \mathrm{~mm} \times 3.7 \mathrm{~mm})$ with reducing edema.

other organs. Our patient responded well to eight-weeks duration of IV meropenem followed by recommended eradication therapy, which include six months of trimethoprim-sulfamethoxazole and folic acid. Repeated brain imaging at three-month interval showed a partial resolution of the basal ganglion abscess (Figure 8) while the repeated brain imaging at six-month intervals showed complete resolution of the ring enhancing lesion in the right basal ganglion and splenic micro- abscesses (image not shown) with full recovery of neurological deficits suggest of good treatment response. His basal ganglion abscess and subdural effusion were not drained as recommended by the neurosurgery team due to its location and difficulty in surgical approach. He is currently under our outpatient follow up for the surveillance of reactivation of the disease.

\section{Learning Points}

The common clinical manifestations of melioidosis infection are pneumonia and localized skin involvement. Culture remains the mainstay of the diagnosis with Gram stain revealing gram-negative bacilli of $B$. pseudomallei. Melioidosis presenting as cerebral abscesses carries a high mortality rate. When encephalomyelitis or brain abscesses are suspected, MRI brain is helpful to delineate the CNS involvement [9]. Prolonged course of antibiotic treatment is indicated in complicated infection and eradication therapy is needed as $B$. pseudomallei have the potential to reactivate, with a latent period that can last up to decades.

\section{Patient's Consent}

Explicit consent from the patient illustrated in this case had been obtained for the medical details and radiographic images to be used for publication purposes.

\section{Acknowledgements}

We thank Dr Rafidah Binti Atan and Dr Naganathan Pillai for their comments that have greatly improved the manuscripts. 


\section{References}

[1] Cheng, A.C. and Currie, B.J. (2005) Melioidosis: Epidemiology, Pathophysiology, and Management. Clinical Microbiology Reviews, 18, 383-416. https://doi.org/10.1128/CMR.18.2.383-416.2005

[2] Currie, B.J., Ward, L. and Cheng, A.C. (2010) The Epidemiology and Clinical Spectrum of Melioidosis: 540 Cases from the 20 Year Darwin Prospective Study. PLOS Neglected Tropical Diseases, 4, e900. https://doi.org/10.1371/journal.pntd.0000900

[3] Currie, B. (2014) Melioidosis: The 2014 Revised RDH Guideline. The Northern Territory Disease Control Bulletin, 21, 4-8.

[4] Wiersinga, W.J., Currie, B.J. and Peacock, S.J. (20120 Melioidosis. The New England Journal of Medicine, 367, 1035-1044. https://doi.org/10.1056/NEJMra1204699

[5] Muttarak, M., et al. (2009) Spectrum of Imaging Findings in Melioidosis. The British Journal of Radiology, 82, 514-521. https://doi.org/10.1259/bjr/15785231

[6] Tung, G.A., et al. (2001) Diffusion-Weighted MR Imaging of Rim-Enhancing Brain Masses: Is Markedly Decreased Water Diffusion Specific for Brain Abscess? American Journal of Roentgenology, 177, 709-712. https://doi.org/10.2214/ajr.177.3.1770709

[7] Woods 2nd, M.L., et al. (1992) Neurological Melioidosis: Seven Cases from the Northern Territory of Australia. Clinical Infectious Diseases, 15, 163-169. https://doi.org/10.1093/clinids/15.1.163

[8] Currie, B.J., et al. (2000) Neurological Melioidosis. Acta Tropica, 74, 145-151. https://doi.org/10.1016/S0001-706X(99)00064-9

[9] Padiglione, A., et al. (1998) Brain Abscesses Caused by Burkholderia pseudomallei. Journal of Infection, 36, 335-337. https://doi.org/10.1016/S0163-4453(98)94639-4

[10] Kilic, O., et al. (2012) Primary Intracranial Abscess Localized in the Basal Ganglia: A Case Report. Journal of Tropical Pediatrics, 58, 71-73. https://doi.org/10.1093/tropej/fmr019

[11] Lutz, T.W., et al. (1994) Diagnosis and Management of Abscesses in the Basal Ganglia and Thalamus: A Survey. Acta Neurochirurgica (Wien), 127, 91-98. https://doi.org/10.1007/BF01808554

[12] Hartmann, M., et al. (2001) Restricted Diffusion within Ring Enhancement Is Not Pathognomonic for Brain Abscess. American Journal of Neuroradiology, 22, 17381742. 
Submit or recommend next manuscript to OALib Journal and we will provide best service for you:

- Publication frequency: Monthly

- 9 subject areas of science, technology and medicine

- Fair and rigorous peer-review system

- Fast publication process

- Article promotion in various social networking sites (LinkedIn, Facebook, Twitter, etc.)

- Maximum dissemination of your research work

Submit Your Paper Online: Click Here to Submit

Or Contact service@oalib.com 